\title{
Latencia y banco de semillas en plantas de la región mediterránea de Chile central
}

\author{
Seed bank and dormancy in plants of the Mediterranean region of central Chile
}

JAVIER A. FIGUEROA \& FABIAN M. JAKSIC

Centro de Estudios Avanzados en Ecología \& Biodiversidad,

Pontificia Universidad Católica de Chile, Casilla 114-D, Santiago CP 6513677, Chile; e-mail: jfiguero@bio.puc.cl

\begin{abstract}
RESUMEN
Los bancos de semillas en las regiones mediterráneas presentan rasgos particulares que los distinguen de bancos de otras regiones del mundo. El banco de semillas es concentración de propágulos viables enterrados en el suelo por períodos variables de tiempo. Los bancos de semillas del matorral de Chile central son principalmente transitorios y funcionalmente semejantes a los bancos presentes en otros ecosistemas mediterráneos no perturbados por fuego, a excepción de Australia. El banco transitorio es aquel formado por semillas que permanecen viables en los horizontes superiores del suelo por no más de 1 año después de la dispersión. En el matorral de Chile central, en los horizontes superiores del suelo, se encuentra una de las densidades de semillas de hierbas más altas de las regiones mediterráneas. Una gran proporción de esta reserva está constituida por semillas sin latencia, fácilmente germinables, especialmente pastos anuales que germinan en otoño. Con respecto a los mecanismos de latencia de las semillas del banco transitorio responsables del almacenamiento de las semillas en el suelo, nuestra revisión bibliográfica reveló los siguientes principales síndromes de germinación: (1) especies con semillas que deben ser estratificadas en frío debido a la presencia de latencia fisiológica (principalmente hierbas introducidas); (2) especies con semillas que deben ser escarificadas con ácidos o procedimientos mecánicos debido a la presencia de latencia física (principalmente especies leñosas nativas); (3) especies con latencia fisiológica que necesitan de estratificación cálida para activar la germinación (hierbas nativas e introducidas). Estos síndromes provocan que la época de germinación en Chile central esté situada entre otoño e inicio de la primavera. Además, no hay referencias sobre la presencia de algún síndrome esporádico de germinación para los componentes de un banco de semillas permanente. Proponemos un modelo estructural y funcional del banco de semillas para el matorral de Chile central, que debería ser enriquecido con futuras investigaciones.
\end{abstract}

Palabras clave: semillas en el suelo, estrategias de germinación, requerimientos de germinación, retraso de germinación, período de germinación.

\begin{abstract}
Seed banks on Mediterranean regions display characteristics that distinguish them from the seed banks of other regions. A seed bank is the concentration of viable propagules buried in the soil for variable periods of time. Seed banks in the Chilean matorral are chiefly transient and functionally similar to seed banks in other Mediterranean ecosystems not disturbed by fire, except for those of Australia. A transient seed bank is that composed of seeds that cannot survive in the upper levels of the soil for more than 1 year. The density of herb seeds in the Chilean matorral soil is one of the highest among Mediterranean ecosystems anywhere in the world, and a large proportion of these seeds is made up of seeds without dormancy that germinate easily, particularly annual grasses. With respect to the dormancy mechanisms that may be responsible for the behavior of these seeds stored in the transient bank, our analysis revealed the following germination syndromes: (1) species whose seeds must be stratified in cold due to the presence of physiological dormancy (particularly introduced herbs), (2) species whose seeds must be scarified with acids or mechanical means due to the presence of physical dormancy (mainly native woody species) and, (3) species that present physiological dormancy and need heat stratification to activate germination (both native and introduced species). These syndromes determine that the timing of germination in central Chile has an autumn and an early spring phase. Besides, there is absence of sporadic germination syndromes for the components of a persistent seed bank. We propose a structural and functional model of the seed bank for the matorral of central Chile that should be enriched with future research.
\end{abstract}

Key words: seeds in soil, germination strategies, germination requirements, germination delay, germination period. 


\section{INTRODUCCIÓN}

Los estudios en banco de semillas y germinación en ecosistemas mediterráneos son numerosos (Baskin \& Baskin 1998). Los bancos de semillas y la biología de ellas presentan rasgos particulares en regiones mediterráneas que los distinguen de bancos de otras regiones del planeta. No obstante, los estudios que han descrito y explicado la estructura, dinámica y función de los bancos de semillas en Chile central son escasos (Fuentes et al. 1984, Jiménez \& Armesto 1992, Jaksic 2001, Figueroa et al. en prensa). Existen algunos estudios de largo plazo en bancos de semillas realizados en regiones con clima árido y semiárido más al norte de la región de Chile central considerada (Gutiérrez et al. 2000, Gutiérrez 2001, Gutiérrez \& Meserve 2003). Además, hay trabajos específicos de corta duración realizados en sitios de alta montaña en los Andes de Chile central (Arroyo et al. 1999, Cavieres \& Arroyo 2001). Otros estudios de bancos de semillas en Chile central están dirigidos principalmente a describir e incrementar la eficiencia del manejo de praderas naturalizadas (Johnston et al. 1991 ${ }^{1}$, Olivares et al. 1994, Olivares et al. $1995^{2}$ ), pero son sitios que difieren en composición y estructura con los matorrales y bosques de baja intervención en Chile central. Con respecto a la biología de la germinación, la gran mayoría de los trabajos existentes tienen el objetivo de manejar y propagar plantas de interés comercial o para la conservación (Donoso \& Cabello 1978, Cabello 1990). Hay pocos estudios de germinación basados en un marco de teoría ecológica en plantas de Chile central.

Esta revisión tiene el objeto de reunir, clasificar y jerarquizar la información disponible en la literatura científica y técnica sobre banco y latencia de semillas en el matorral esclerófilo siempreverde de Chile central. Con esta información se propone un modelo conceptual de los bancos de semillas para esta región. Un objetivo secundario es generalizar sobre las funciones y estructuras de los bancos de semillas de clima mediterráneo derivadas de la comparación entre Chile central y otros ecosistemas similares en el mundo (Baskin \& Baskin 1998).

\footnotetext{
1 JOHNSTON M, A OLIVARES \& G FERNÁNDEZ (1991) Soil seed-bank of an annual Mediterranean grassland. Fourth International Rangeland Congress, Montpellier, France.

2 OLIVARES A, M JOHNSTON \& X CONTRERAS (1995) Interaction between arboreous stratum, management system of annual grassland and soil-seed reserve. Fifth International Rangeland Congress, Utah, United States of America.
}

REGIÓN MEDITERRÁNEA DE CHILE CENTRAL

\section{Clima}

Los ecosistemas de tipo mediterráneo se caracterizan por veranos cálidos y secos e inviernos fríos y lluviosos. Este clima es compartido por cinco áreas geográficas del mundo: California, Chile central, Cuenca del Mar Mediterráneo, Sudáfrica y Australia. En Chile central el clima mediterráneo se extiende desde $31^{\circ}$ hasta $37^{\circ} \mathrm{S}$ y se caracteriza por presentar dos estaciones dicotómicas. La estación cálida y seca transcurre durante el verano que en la zona media de la región se extiende entre octubre y abril. Además, entre diciembre y febrero se presentan las mayores diferencias entre la temperatura máxima promedio y la temperatura mínima promedio $\left(16-17{ }^{\circ} \mathrm{C}\right)$ y precipita alrededor del $3 \%$ del total de lluvia anual (Jaksic 2001). La estación de invierno es fría y lluviosa y se extiende desde mayo a septiembre (di Castri \& Hajek 1976) con variables patrones interanuales de precipitación (Aceituno 1990). Entre mayo y agosto se presentan las menores diferencias entre la temperatura máxima y mínima promedio $\left(9-10^{\circ} \mathrm{C}\right)$.

En las mismas coordenadas geográficas de Chile central, sobre los $2.000 \mathrm{~m}$ de altitud persiste un clima mediterráneo frío y vegetación de tipo alto andino que será excluido de esta revisión. De la misma manera serán excluidos los sistemas áridos y semiáridos inmediatamente al norte de Chile central. Esta decisión se tomó debido a las características particulares de la flora, dinámica y función en los bancos de semillas de ambas regiones (Vidiella \& Armesto 1989, Arroyo et al. 1999, Cavieres 1999, Gutiérrez et al. 2000, Gutiérrez 2001, Gutiérrez \& Meserve 2003) y principalmente porque nuestra revisión está concentrada en el matorral y bosque esclerófilo siempreverde de Chile central.

\section{Vegetación en Chile central}

Esta es una región con una notable diversidad florística (Myers et al. 2000) debido a que la vegetación se extiende sobre un relieve complejo y heterogéneo, dentro de un área de transición climática que presentó condiciones inestables durante el Cuaternario (Villagrán 1995, Cowling et al. 1996). La vegetación de Chile central comprende principalmente matorral y bosque esclerófilo de baja altura compuesto de parches de flora leñosa de composición y edad heterogéneas (Fuentes et al. 1984). Esta vegetación se extiende desde los $32^{\circ}$ hasta los $36^{\circ} \mathrm{S}$ (Gajardo 1994). El matorral está constituido principalmente por arbustos bajos espinosos y xerofíticos con hojas 
esclerófilas. Las especies leñosas están acompañadas por una diversa flora herbácea dominada por especies anuales introducidas y nativas (Arroyo et al. 2000). Actualmente, esta vegetación está sometida a una fuerte presión por extracción de leña, habilitación de tierra para agricultura y vivienda, ramoneo por animales domésticos y fuego de origen antrópico (Fuentes et al. 1986, Fuentes 1990, Armesto et al. 1995, Aronson et al. 1998, Montenegro et al. 2002). Existen estudios que han evaluado el efecto directo e indirecto de la alteración antropogénica sobre la composición y dinámica del banco de semillas de especies leñosas en el matorral (Fuentes et al. 1984, Jiménez \& Armesto 1992) o sobre especies anuales en praderas manejadas (Olivares et al. 1994) de Chile central. Sin embargo, es poco lo que se conoce respecto de la distribución y composición del banco de semillas de especies herbáceas en el matorral de esta región (Keeley \& Johnson 1977, Jaksic 2001, Figueroa et al. en prensa).

En Chile central no existe comunidad vegetal que no haya experimentado algún tipo de influencia antrópica. Por lo tanto, la composición y dinámica actual del estrato herbáceo y leñoso del matorral es el resultado de la acción directa o indirecta del hombre sobre las comunidades de plantas originarias (Holmgren 2002). Uno de los efectos más notorios de estas perturbaciones sobre las comunidades es la alta diversidad y concentración de flora adventicia e introducida naturalizada (Montenegro et al. 1991, Arroyo et al. 2000). Esta revisión incorporará a las especies introducidas y naturalizadas en Chile central como un componente estructural y funcional del actual matorral, debido a que es altamente improbable que los sistemas actuales vuelvan a su condición original (Holmgren 2002) y a la importancia actual de la flora exótica naturalizada en el matorral mediterráneo de Chile central. Como veremos posteriormente, la flora introducida no solo se encuentra establecida junto a la flora nativa sino que tiene un papel muy importante en la estructura y función de las reservas de semillas en el suelo. Esta es la primera revisión de estudios funcionales en plantas que incorpora de manera explícita las especies de hierbas introducidas como elementos estructurales de las comunidades de plantas en Chile central.

\section{BANCO DE SEMILLAS}

El banco de semillas es la concentración de propágulos viables enterrados en el suelo por períodos variables de tiempo (Thompson \&
Grime 1979). En el banco de semillas pueden ser distinguidas dos fracciones, de acuerdo al tiempo que permanecen viables los propágulos en el suelo. La fracción de semillas enterradas principalmente en los horizontes superiores del suelo $(<5 \mathrm{~cm}$ de la superficie) y que no permanece más de 1 año viable sin germinar se denomina banco de semillas transitorio (Thompson \& Grime 1979). Por otra parte, aquella fracción del banco que permanece por más de 1 año enterrado y viable principalmente en los horizontes más profundos (> $5 \mathrm{~cm})$, se denomina banco de semillas persistente (Thompson \& Grime 1979). Además, en el suelo puede encontrarse un acervo de semillas sin latencia, fácilmente germinables que es mantenido por ambas fracciones del banco de semillas y que origina nuevas plántulas para el establecimiento. En muchas poblaciones de plantas mediterráneas el banco de semillas es la principal reserva de los nuevos reclutas y en gran parte determina la composición futura de la comunidad de plantas establecidas (Parker \& Kelly 1989).

Sin embargo, los estudios que se han realizado en el matorral de Chile central sugieren que el banco de las especies leñosas bajo el suelo sería muy pobre y estarían ausentes los principales elementos que se encuentran en la vegetación establecida (Muñoz \& Fuentes 1989, Armesto et al. 1995). Por ejemplo, Cryptocarya alba, una especie dominante del matorral esclerófilo en la cordillera de la Costa de Chile central, no se registró ni en el banco de semillas bajo el suelo del matorral ni en los campos abandonados (Jiménez \& Armesto 1992). Incluso, en los sitios estudiados por Jiménez \& Armesto (1992) solo ocho especies leñosas fueron registradas en el banco de semillas y todas ellas con densidades menores a 200 semillas $\mathrm{m}^{-2}$ (Armesto et al. 1995). Además, los ensayos de germinación mostraron que ninguna semilla encontrada germinó desde las muestras de suelo obtenidas en los sitios abiertos entre arbustos y en los campos abandonados. Las semillas de $C$. alba solo germinaron desde las muestras de suelo del matorral denso y en muy baja proporción (Jiménez \& Armesto 1992). Igualmente, una densidad baja de semillas de especies leñosas $\left(<50\right.$ semillas $\mathrm{m}^{-}$ ${ }^{2}$ ) fue registrada en un matorral ubicado al pie de la cordillera de los Andes (Figueroa et al. en prensa). Estas densidades en el banco de semillas en el suelo del matorral de Chile central son, por ejemplo, notoriamente más bajas que las registradas para especies leñosas en los bancos del matorral abierto de California con densidades superiores a 1.000 semillas $\mathrm{m}^{-2}$ para las especies dominantes (Parker \& Kelly 1989). Las especies leñosas de los fynbos de Sudáfrica también pre- 
sentan bajas densidades de semillas almacenadas en el suelo (Pierce \& Cowling 1991) o su contribución es menor que las especies de hierbas (Holmes \& Cowling 1997). Una situación semejante es lo que ocurre en la vegetación esclerófila de Australia (Parker \& Kelly 1989). Tanto en Sudáfrica como en Australia, muchas especies leñosas tienen, por el contrario, banco de semillas persistente sobre el dosel (especies serotinas), hasta que el fuego induce la dispersión de las semillas por gravedad, viento o dehiscencia de los frutos secos y la germinación se inicia después de la dispersión de las semillas (Keeley 1995, Vázquez-Yanes \& OrozcoSegovia 1998). En resumen, las evidencias sugieren que los bancos de semillas de especies leñosas de los matorrales de California y mediterráneos de Europa (Parker \& Kelly 1989, Holmes \& Cowling 1997, Valbuena \& Trabaud 2001) son los más abundantes y persistentes de todos los bancos de las regiones con climas de tipo mediterráneo. Por el contrario, los bancos de semillas de la vegetación leñosa de Chile central, se encuentran entre los más pobres en semillas de las regiones mediterráneas (Jiménez \& Armesto 1992), aunque en el matorral de Chile central no se conocen especies serotinas. En el matorral, donde el fuego es generalmente antropogénico (Aronson et al. 1998), muchas especies leñosas son capaces de persistir a los incendios debido a la presencia de tallos y raíces que rebrotan después de fuegos (Ginocchio et al. 1994, Montenegro et al. 2002).

Por otra parte, la composición de las hierbas del matorral de Chile central, que está dominada fuertemente por especies anuales introducidas, es diversa y abundante (Gulmon 1977, Groves 1986, Montenegro et al. 1991, Jaksic 2001). Empero, el estudio del banco de semillas de las especies de hierbas ha sido postergado por muchos años. Antecedentes recientes de un estudio realizado por Figueroa et al. (en prensa) en San Carlos de Apoquindo (precordillera de Santiago, $33^{\circ} \mathrm{S}$ ), sugieren que la composición del banco de semillas sin latencia, fácilmente germinable, está constituido principalmente por hierbas anuales. Este banco de semillas involucra alrededor de 60 especies de plantas, siendo las anuales las más abundantes (46 especies). No obstante, el banco está dominado por solo cinco especies de hierbas que representan $>80 \%$ de la densidad total de semillas en la época de verano (la de mayor acumulación de semillas): la especie anual nativa Bromus berterianus, las introducidas Anthriscus caucalis y Vulpia bromoides, y las perennes nativas Gamochaetha stachydifolia y Gnaphalium philippi.
Figueroa et al. (en prensa) encontraron que el banco de semillas de las especies de hierbas en el matorral de Chile central, a diferencia de las semillas de especies leñosas, es un componente de la comunidad altamente dinámico. El factor que más contribuyó a la variación de la densidad y riqueza florística del banco de semillas fue, en primer lugar, el efecto de la etapa del ciclo de vida en que se hallaban las especies anuales dominantes. Por ejemplo, los pastos anuales acumularon en el suelo por lo menos 6.000 semillas $\mathrm{m}^{-2}$ al término de su época de fructificación. En contraste, la densidad de los pastos anuales en el banco del suelo alcanzó solo 100 semillas $\mathrm{m}^{-2}$ al término de la época de germinación, que coincide con el inicio de la primavera.

Por otro lado, la variación espacial del banco fue menos extensa y significativa (Figueroa et al. en prensa). Sin embargo, la disminución de la densidad de semillas fue dramática bajo el dosel continuo de la sucesión madura, dominada por Quillaja saponaria y Lithrea caustica, cuyos individuos alcanzaban $>3 \mathrm{~m}$ de alto. Estos mismos investigadores encontraron que en dichos hábitats se registraron solamente 17 semillas $\mathrm{m}^{-2}$ de pastos. Por el contrario, fuera del dosel, en la época de mayor concentración de semillas en el suelo, la densidad registraba alrededor de 6.000 semillas $\mathrm{m}^{-2}$, independientemente de si las muestras fueron obtenidas en parches de sucesión temprana o intermedia. Figueroa et al. (en prensa) sugieren que hay una dispersión limitada de semillas de gramíneas hacia la sucesión madura, debido a que bajo el dosel continuo de los árboles no hay establecimiento de pastos ni sus propágulos están almacenados en el suelo.

Por otra parte, la concentración de semillas enterradas en el suelo del matorral de Chile central con baja perturbación antrópica es notoriamente menor a la registrada en el suelo de praderas anuales sometidas a pastoreo y dominadas por gramíneas; para las cuales se determinaron entre 15.000 y 35.000 semillas $\mathrm{m}^{-2}$ (Olivares et al. 1994).

Las evidencias de la literatura muestran que la densidad de semillas de hierbas almacenadas en el suelo del matorral mantiene una de las densidades más altas en las regiones de tipo mediterráneo, a excepción de California (Parker \& Kelly 1989). En los fynbos de Sudáfrica, la densidad de semillas de hierbas dominantes enterradas en el suelo se ubica entre 1.000 y 5.000 semillas $\mathrm{m}^{-2}$ (Holmes \& Cowling 1997). Por otra parte, la riqueza de especies de hierbas en el banco de semillas de los fynbos de Sudáfrica es menor a la riqueza del matorral de Chi- 
le central y tiene representado alrededor del 60 $\%$ del total de las especies establecidas. En los fynbos, las especies dominantes, en relación con la densidad de semillas, representan sobre el $90 \%$ del total de semillas del suelo. Finalmente, en el Mediterráneo de España las especies de hierbas representan más del $80 \%$ del total de especies registradas en el banco (Valbuena \& Trabaud 2001). Además, en esta última región la densidad de semillas de hierbas es de 1.000 semillas $\mathrm{m}^{-2}$ aproximadamente.

En resumen, la función del banco de semillas en el suelo en el matorral de Chile central parece ser semejante a aquellos de los suelos no perturbados por fuego del chaparral de California (Parker \& Kelly 1989), praderas de la cuenca del Mar Mediterráneo (Peco et al. 1998, Russi et al. 1992) y de Chile central (Olivares et al. 1994), en los que predominan las semillas transitorias. Asimismo, las evidencias sugieren que en Chile central las semillas de los pastos anuales dominantes Bromus sp. y Vulpia sp. se dispersarían, principalmente sin latencia para constituir, inmediatamente después de la dispersión de los propágulos, el acervo de semillas fácilmente germinable (Figueroa et al. en prensa). Ambas taxa germinarían con el inicio de las lluvias de otoño. Además, las especies de Erodium, taxón introducido común en el mediterráneo de Chile, diseminan sus semillas durante la primavera con una latencia que es interrumpida por la escarificación natural y las bajas temperaturas del invierno siguiente a su dispersión (Johnston et al. 1989). Es decir, las semillas de Erodium, que germinan al inicio de otoño, no pasarían más de 2 años en el suelo de la vegetación de Chile central y este tiempo disminuiría con un incremento de las precipitaciones anuales (Johnston et al. 19933).

\section{LONGEVIDAD DE LAS SEMILLAS}

Para que las semillas puedan permanecer almacenadas en el suelo deben presentar propiedades que les permitan mantener su viabilidad bajo condiciones variables del ambiente y algún tipo de latencia. Se han definido como semillas ortodoxas a aquellas que pueden permanecer viables bajo condiciones controladas de almacenamiento (Roberts 1973). Debido a que

\footnotetext{
3 JOHNSTON M, A OLIVARES \& G FERNÁNDEZ (1993) An investigation into the effect of some factors on the germination of Erodium seeds. Proceedings of the XVII International Grassland Congress, Palmerston North, New Zealand.
}

una proporción importante de las especies que habitan ambientes de tipo mediterráneo presentan una testa dura e impermeable, se ha sugerido que las especies de estos ecosistemas producen semillas de tipo ortodoxo (Vázquez-Yanes \& Orozco-Segovia 1998). Incluso, hay modelos corroborados con investigaciones de campo, que sugieren que este comportamiento y la latencia prolongada serían comunes en especies que presentan semillas pequeñas y con breves ciclos de vida (Venable \& Lawlor 1980, Venable 1989, Rees 1993). Hay evidencias de un comportamiento ortodoxo en semillas pequeñas de la especie anual nativa Bromus berterianus y en las introducidas Hordeum murinum, Erodium malacoides, Medicago sp y Vulpia bromoides (Johnston et al. 1992), comunes en la vegetación de matorral.

Por el contrario, taxa de géneros de origen neotropical presentes en Chile central, que producen semillas relativamente grandes, tienen un comportamiento de tipo recalcitrante. Este comportamiento se refiere a semillas que no pueden ser almacenadas porque pierden rápidamente su viabilidad cuando son deshidratadas y mantenidas a bajas temperaturas (Roberts 1973) y, por lo general, no presentan latencia o ella es débil. Entre otras, las lumas presentes en Chile central pierden su viabilidad a las pocas semanas de estar almacenadas debido a que poseen embriones desnudos (Landrum 1981) con un alto contenido hídrico (Ramírez et al. 1980, Figueroa \& Armesto 2001). Hay evidencias de semillas con un comportamiento recalcitrante en Luma chequen, L. apiculata, Myrceugenia obtusa, Bielschmiedia berteroana, Citronella mucronata, Cryptocarya alba (Cabello 1990, Bustamante et al. 1996) y en la especie anual introducida Avena barbata (Johnston et al. 1992). Sin embargo, no hay estudios experimentales que determinen las condiciones térmicas e hídricas óptimas para almacenar propágulos viables por períodos prolongados de tiempo en especies de la vegetación de Chile central (León-Lobos, comunicación personal).

\section{MECANISMOS DE LATENCIA}

El impedimento de la germinación de semillas viables bajo condiciones ambientales adecuadas es provocado por mecanismos de latencia innata o latencia inducida por factores ambientales. Para que ocurra la germinación, las semillas no deben estar en un estado de latencia y las condiciones ambientales para la germinación deben ser las apropiadas (Baskin \& Baskin 1989). La latencia de las semillas tendría una función 
adaptativa, debido a que es un mecanismo que evita que las pequeñas plántulas emerjan desde el suelo cuando las condiciones ambientales son inadecuadas para su establecimiento exitoso (Figueroa \& Armesto 2001). La estación de verano en las regiones con clima mediterráneo es la de mayor estrés, condición que dificulta el establecimiento de plántulas debido a la ausencia de precipitaciones relevantes. No obstante, un número importante de especies del matorral fructifica y dispersa sus propágulos durante los meses de verano (Hoffmann et al. 1989). Por lo tanto, algún mecanismo de latencia, para el momento de la dispersión de las semillas, garantizaría que el número de semillas germinadas sea irrelevante para los meses estivales (VázquezYanes \& Orozco-Segovia 1998). En este contexto, en Chile central hay pocas evidencias experimentales de que especies nativas produzcan semillas sin latencia para el mismo momento de la dispersión. Sin embargo, una excepción es el pasto anual nativo Bromus berterianus, que presenta semillas que germinan rápidamente con las primeras lluvias del otoño (Johnston et al. 1989).

Existen antecedentes de que el impedimento más frecuente de la germinación para especies leñosas nativas del matorral es la latencia física provocada por una testa dura e impermeable. La cubierta de la semilla llega a ser permeable naturalmente con el paso del tiempo, a través de escarificación natural, calor, fuego y la acción de ácidos que corroen la testa (MohamedYasseen et al. 1994). Para Chile central, hay antecedentes de latencia física en Acacia caven, Adesmia microphylla, Cassia closiana, C. tomentosa, Lithrea caustica, Retamilla ephedra, Retamilla trinervis y Trevoa quinquenervia (Hoffmann \& Kummerow 1962, Donoso \& Cabello 1978, Cabello 1979, Muñoz \& Pérez 1981). La mayoría de estas especies pueden ser estimuladas a germinar con aplicación de ácidos sobre la testa de las semillas o una escarificación mecánica. La consecuencia inmediata de este mecanismo de latencia física sería el retraso de la germinación en las especies que dispersan sus semillas al inicio o durante la extensa sequía de verano.

Además, una latencia fisiológica de corta duración ha sido detectada para algunas especies leñosas nativas del matorral, la que es liberada por la acción de bajas temperaturas, similares a las exhibidas durante el invierno de la región. El enfriamiento de las semillas puede reducir la latencia a través de la movilización de las reservas de recursos (Bell 1999). Este mecanismo retrasa la germinación y el establecimiento hasta finales del invierno e inicio de la primavera, respectivamente, cuando en Chile central las condiciones para el establecimiento son más favorables. Especies con estas características son Colletia spinosa, Crinodendron patagua, Austrocedrus chilensis y Rhaphithamnus spinosus (Donoso \& Cabello 1978, Cabello 1990, Figueroa et al. 1996). Otro grupo más pequeño de especies nativas con latencia fisiológica presenta el síndrome opuesto. Ellas producen semillas que requieren un período de elevadas temperaturas para activar la germinación, similares a las exhibidas durante el verano de la región. Este mecanismo permite que el establecimiento de las plántulas se inicie con el otoño. Las temperaturas cálidas pueden reducir la latencia a través de inactivar inhibidores de germinación o alterar la respiración celular (Bell 1999). Por ejemplo, presentan este síndrome Jubaea chilensis (Infante 1989), Peumus boldus (Cabello \& Infante $1986^{4}$ ) y varias especies de Alstroemeria (Thompson et al. 1979).

Las evidencias indican que en especies del matorral de Chile central el fuego no induciría la germinación (Muñoz \& Fuentes 1989), a diferencia de California (Keeley 1995), región Mediterránea (Naveh 1975), Sudáfrica (Boucher \& Moll 1981) y Australia (Parsons 1981). Sin embargo, Colliguaya odorifera y Muehlenbeckia hastulata incrementaron notoriamente su germinación de semillas cuando se les aplicó un tratamiento breve de temperatura alta (Muñoz \& Fuentes 1989). En estas especies del matorral, el calor puede provocar un efecto similar a la acción del ácido sulfúrico sobre la testa (Muñoz \& Fuentes 1989). El calor puede provocar la abrasión de la cubierta de la semilla en las especies que presentan testa dura e impermeable o la destrucción de un inhibidor químico (Bell 1999). Además, los componentes químicos del humo del fuego pueden también estimular la germinación (Bell 1999), indicando la existencia de un sensor en las semillas que no ha sido aún identificado (Keeley \& Fotheringham 1997, Van Staden et al. 2000). El efecto del humo a temperatura ambiental fue evaluado recientemente en 18 especies leñosas de Chile central (Cavieres, comunicación personal). Los resultados mostraron que el humo estimuló la germinación de tres especies (Acacia caven, Baccharis vernalis y Trevoa quinquenervia) e inhibió otras ocho, varias de las

\footnotetext{
${ }^{4}$ CABELLO A \& L INFANTE (1986) Ensayos preliminares de germinación en palma chilena (Jubaea chilensis (Mol.) Baillón). Sexta Reunión Nacional de Botánica, Universidad Austral de Chile, Valdivia, Chile.
} 
cuales son nativas dominantes del matorral (e.g., Lithrea caustica, Quillaja saponaria, Cryptocarya alba).

La flora leñosa del matorral de Chile central presenta una frecuencia importante de especies con frutos carnosos (Hoffmann et al. 1989, Hoffmann \& Armesto 1995). El tejido carnoso que rodea la semilla puede inducir una latencia ejercida por inhibidores químicos de la germinación que podrían encontrarse en la pulpa $(\mathrm{Ci}-$ pollini \& Levey 1997). Por lo tanto, animales que ingieren frutos y dispersan semillas pueden facilitar la germinación (Bell 1999). Asimismo, el efecto inductor de germinación por la ingestión de semillas en zorros y aves frugívoras ha sido detectado en varias especies leñosas nativas de Chile central. La digestión de frutos por zorros estimuló la germinación de semillas de Cryptocarya alba (Bustamante et al. 1992), Lithrea caustica (León-Lobos \& Kalin-Arroyo 1994), Schinus molle (Castro et al. 1994) y Porlieria chilensis (Silva 2001). El consumo de pericarpo por aves incrementó la germinación en Cryptocarya alba (Bustamante et al. 1996) y la digestión por tencas estimuló la germinación de Tristerix aphyllus (Soto-Gamboa \& Bozinovic 2002). La extracción manual de pericarpo estimuló la germinación de semillas de Beilschmiedia berteroana, Cryptocarya alba, Geoffroea decorticans, Persea lingue y la extracción del arilo estimuló la germinación de Maytenus boaria (Cabello 1990), sugiriendo la presencia de algún inhibidor químico.

Por otra parte, las especies de Alstroemeria, Beilschmiedia miersii, Maytenus boaria y Myrceugenia correifolia, se asemejan porque presentan bajas temperaturas óptimas de germinación de semillas (Thompson et al. 1979, Cabello \& Camelio 1995, Cabello et al. 2000). Esta parece ser una característica generalizada de las especies mediterráneas (Vázquez-Yanes \& Orozco-Segovia 1998), aunque ello no es corroborado por registros de temperatura óptima sobre los $20{ }^{\circ} \mathrm{C}$ para muchas especies exóticas de Chile central (Tabla 1).

Con respecto a las especies introducidas y naturalizadas en Chile central, que en su mayoría son anuales y de origen euroasiático, mayoritariamente presentan semillas con latencia fisiológica, que debe ser interrumpida por estratificación fría (e.g., Chenopodium album, Digitaria sanguinalis, Plantago lanceolata, Poa pratensis) o estratificación cálida (e.g., Avena fatua, Poa annua, Portulaca oleracea, Stellaria media) (Tabla 1). Un grupo menos numeroso de anuales introducidas presenta latencia de tipo física, que debe ser interrumpida por escarificación de la testa (e.g., Cyperus rotun- dus, Erodium spp., Ipomoea purpurea) (Tabla 1). Sin embargo, no es despreciable el número de anuales introducidas, que tienen semillas que no presentan mecanismos de latencia para el momento de la dispersión (e.g., Cardus nutans, Hordeum murinum, Lactuca serriola, Vulpia bromoides) (Tabla 1).

A modo de comparación, el mecanismo de latencia más frecuente en vegetación leñosa de regiones mediterráneas parece ser la testa dura e impermeable de las semillas o latencia de tipo física (Baskin \& Baskin 1998, Vázquez-Yanes \& Orozco-Segovia 1998). En Australia, muchos arbustos con latencia física pertenecen a las familias Fabaceae y Celastraceae; en California y Chile varios pertenecen a Rhamnaceae y en la cuenca del Mediterráneo a Cistaceae. No obstante el escaso conocimiento que existe sobre germinación de hierbas nativas del matorral, las pocas evidencias en la literatura sugieren una mayor importancia de latencias de tipo fisiológica (Tabla 1).

Todos los mecanismos de latencia interactúan entre ellos y con una variedad de factores ambientales, provocando una gran diversidad de respuestas de germinación adecuadas a las diferentes perturbaciones y estaciones de un clima mediterráneo. Concordamos con Vázquez-Yanes \& Orozco-Segovia (1998) en que esto puede ser el origen del escaso banco de semillas persistente formado por semillas de cubierta delgada en diferentes áreas de clima de tipo mediterráneo. Sin embargo, aún se desconoce mucho de los mecanismos de latencia y requerimientos de germinación en el matorral de Chile central, especialmente en hierbas nativas, comparado al extenso conocimiento en otras regiones de clima mediterráneo (Bakin \& Baskin 1998).

\section{CONCLUSIONES}

Para finalizar presentamos un modelo conceptual, que es una variación del modelo de Harper (1977), que sistematiza la información disponible para Chile central. En la zona mediterránea de Chile central hay un acervo dinámico, diverso y concentrado de semillas germinables sin latencia principalmente durante los meses de verano e inicio del otoño. Esta alta concentración de propágulos se debe principalmente al aporte de pastos anuales nativos e introducidos a través de la lluvia de semillas (Fig. 1). Con el inicio de las lluvias en el otoño, el acervo de semillas germinables es evacuado paulatinamente debido a la germinación de pastos que diseminan semillas sin latencia, a la germina- 
TABLA 1

Lista de especies de Chile central con los principales rasgos de germinación considerados

Species list of central Chile with germination features considered

\begin{tabular}{|c|c|c|c|c|c|c|}
\hline Especie & Familia & $\mathrm{L} /$ Trat $^{1}$ & Temp $^{2}$ & $\mathrm{Luz}^{3}$ & Otros $^{4}$ & Referencia \\
\hline Acacia caven & Mimosaceae & Lfis/E & $15-35$ & & & Cabello (1990), Cabello \& Alvear (1999) \\
\hline Adesmia arborea & Papilionaceae & $\mathrm{LN}$ & & & & Cabello (1990) \\
\hline Adesmia microphylla & Papilionaceae & Lfis/E & & & & Cabello (1990) \\
\hline Agrostemma githago & Caryophyllaceae & $\mathrm{LF} / \mathrm{EC}$ & $5-25$ & $L>$ & & Baskin \& Baskin (1998) \\
\hline Alopecurus myosuroides & Poaceae & $\mathrm{LF} / \mathrm{EC}$ & $25 / 15$ & L> & & Baskin \& Baskin (1998) \\
\hline Amsinckia hispida & Boraginaceae & $\mathrm{LF}$ & $12-13$ & & & Baskin \& Baskin (1998) \\
\hline Alstroemeria haemantha & Amarylliaceae & $\mathrm{LF} / \mathrm{EC}$ & 11 & & & Thompson et al. (1979) \\
\hline Alstroemeria pulchra & Amarylliaceae & $\mathrm{LF} / \mathrm{EC}$ & 11 & & & Thompson et al. (1979) \\
\hline Alstroemeria revoluta & Amarylliaceae & $\mathrm{LF} / \mathrm{EC}$ & 11 & & & Thompson et al. (1979) \\
\hline Amaranthus hybridus & Amaranthaceae & $\mathrm{LF} / \mathrm{EF}$ & $35 / 20$ & $L>$ & & Baskin \& Baskin (1987) \\
\hline Amaranthus retroflexus & Amaranthaceae & $\mathrm{LF} / \mathrm{EF}$ & $37 / 20$ & & & Baskin \& Baskin (1998) \\
\hline Ambrosia artemisiifolia & Asteraceae & $\mathrm{LF} / \mathrm{EF}$ & $30 / 15$ & L> & & Baskin \& Baskin (1980) \\
\hline Anagallis arvensis & Primulaceae & LF./EC & $9-12$ & $\mathrm{~L}$ & & Baskin \& Baskin (1998) \\
\hline Anoda cristata & Malvaceae & Lfis & 30 & $=$ & & Solano et al. (1976) \\
\hline Anthemis cotula & Asteraceae & $\mathrm{LF} / \mathrm{EC}$ & 20 & & & Baskin \& Baskin (1998) \\
\hline Anthriscus silvestris & Apiaceae & LMF & $8 / 4$ & & & Baskin \& Baskin (1998) \\
\hline Atriplex patula & Chenopodiacea & $\mathrm{LF} / \mathrm{EF}$ & $20 / 15$ & $L>$ & & Grime et al. (1981) \\
\hline Atriplex repanda & Chenopodiaceae & Lfis/E & & L> & Lav/ $\mathrm{NaCl}$ & $\begin{array}{l}\text { Fernández (1978), } \\
\text { Gutiérrez et al. (1988), Moreno et al. (1990) }\end{array}$ \\
\hline Austrocedrus chilensis & Cupressaceae & $\mathrm{LF} / \mathrm{EF}$ & & & & Pais $(2000)$ \\
\hline Avena barbata & Poaceae & Lfis/E & 10 & & & Johnston et al. (1989) \\
\hline Avena fatua & Poaceae & $\mathrm{LF} / \mathrm{EC}$ & 16,21 & $L>$ & & Baskin \& Baskin (1998) \\
\hline Beilschmiedia berteroana & Lauraceae & $\mathrm{LN}$ & & & Sin pulpa & Cabello (1990) \\
\hline Beilschmiedia miersii & Lauraceae & $\mathrm{LF} / \mathrm{EF}$ & $10-15$ & & & Cabello \& Anselmo (1999) \\
\hline Bidens pilosa & Asteraceae & SL & $25 / 20$ & $L>$ & & Baskin \& Baskin (1998) \\
\hline Bromus berterianus & Poaceae & SL & $20 / 10$ & & & Johnston et al. (1989) \\
\hline Bromus mollis & Poaceae & & 15 & & & Olivares et al. (1990) \\
\hline Bromus secalinus & Poaceae & $\mathrm{LF} / \mathrm{EC}$ & 15 & & & Baskin \& Baskin (1998) \\
\hline Bromus tectorum & Poaceae & $\mathrm{LF} / \mathrm{EC}$ & $14-17$ & & & Baskin \& Baskin (1998) \\
\hline Capsella bursa-pastoris & Brassicaceae & $\mathrm{LF} / \mathrm{EC}$ & $20 / 10$ & L> & & Baskin \& Baskin (1989) \\
\hline Cardus nutans & Asteraceae & SL & $30 / 20$ & $L>$ & & Baskin \& Baskin (1998) \\
\hline Cassia closiana & Leguminosaceae & Lfis/E & & & & Cabello (1990) \\
\hline Cassia tomentosa & Leguminosaceae & Lfis/E & & & & Cabello (1990) \\
\hline Chenopodium album & Chenopodiaceae & $\mathrm{LF} / \mathrm{EF}$ & $30 / 15$ & $L>$ & & Baskin \& Baskin (1998) \\
\hline Citronella mucronata & Icacinaceae & $\mathrm{LN}$ & & & & Cabello (1990) \\
\hline Colletia hystrix & Rhamnaceae & $\mathrm{LF} / \mathrm{EF}$ & & & & Donoso \& Cabello (1978) \\
\hline Colletia spinosa & Rhamnaceae & $\mathrm{LN}$ & & & & Muñoz \& Fuentes (1989) \\
\hline Colliguaja odorifera & Euphorbiaceae & Lfis & & & Golpe $\mathrm{T}^{\circ}$ & Muñoz \& Fuentes (1989) \\
\hline Conium maculatum & Apiaceae & LM & $30 / 15$ & $L>$ & & Baskin \& Baskin (1990a) \\
\hline Convolvulus arvensis & Convolvulaceae & Lfis/E & $35 / 20$ & $=$ & & Baskin \& Baskin (1998) \\
\hline Cotula coronopifolia & Asteraceae & SL & $30 / 15$ & & & Baskin \& Baskin (1998) \\
\hline Crinodendron patagua & Tiliaceae & $\mathrm{LF} / \mathrm{EF}$ & & & & Donoso \& Cabello (1978) \\
\hline Cryptocarya alba & Lauraceae & SL & & & Digestión & Bustamante et al. $(1992,1996)$ \\
\hline Cyperus esculentus & Cyperaceae & $\mathrm{LF} / \mathrm{EF}$ & $38 / 32$ & L> & & Baskin \& Baskin (1998) \\
\hline Cyperus odoratus & Cyperaceae & $\mathrm{LF} / \mathrm{EF}$ & $35 / 20$ & $\mathrm{~L}$ & & Baskin et al. (1989) \\
\hline Cyperus rotundus & Cyperaceae & Lfis/EC & $38 / 32$ & $L>$ & & Baskin \& Baskin (1998) \\
\hline Daucus carota & Apiaceae & $\mathrm{LM} / \mathrm{EF}$ & $30 / 15$ & & & Baskin \& Baskin (1998) \\
\hline Dianthus armeria & Caryophyllaceae & $\mathrm{LF} / \mathrm{EC}$ & $30 / 15$ & $\mathrm{~L}$ & & Baskin \& Baskin (1998) \\
\hline
\end{tabular}


TABLA 1 (Continuación)

\begin{tabular}{|c|c|c|c|c|c|c|}
\hline Especie & Familia & $\mathrm{L} /$ Trat $^{1}$ & Temp $^{2}$ & $\mathrm{Luz}^{3}$ & Otros $^{4}$ & Referencia \\
\hline Digitaria ischaemum & Poaceae & $\mathrm{LF} / \mathrm{EF}$ & $35 / 20$ & L> & & Baskin \& Baskin (1998) \\
\hline Digitaria sanguinalis & Poaceae & $\mathrm{LF} / \mathrm{EF}$ & $35 / 20$ & $\mathrm{~L}>$ & & Baskin \& Baskin (1998) \\
\hline Drimys winteri & Winteraceae & $\mathrm{LM} / \mathrm{EF}$ & & & & Cabello \& Botti (1987) \\
\hline Echinochloa crus-galli & Poaceae & $\mathrm{LF} / \mathrm{EF}$ & $35 / 20$ & $=$ & & Baskin \& Baskin (1998) \\
\hline Emex spinosa & Polygonaceae & LF & $30 / 20$ & $L>$ & & Baskin \& Baskin (1998) \\
\hline Erodium botrys & Geraniaceae & & $5-15$ & & & Gerrero \& Williams (1975) \\
\hline Erodium cicutarium & Geraniaceae & Lfis/E & $20 / 15$ & & & Grime et al. (1981) \\
\hline Erodium malacoides & Geraniaceae & Lfis/E & & & & Johnston et al. (1989) \\
\hline Erodium moschatum & Geraniaceae & Lfis/E & & & & Johnston et al. (1989) \\
\hline Escallonia pulverulenta & Saxifragaceae & SL & & & & Cabello (1990) \\
\hline Euphorbia maculata & Euphorbiaceae & $\mathrm{LF} / \mathrm{EF}$ & $35 / 20$ & $L>$ & & Baskin \& Baskin (1979a) \\
\hline Fumaria officinalis & Fumariaceae & $\mathrm{LMF} / \mathrm{EF}$ & 20 & & & Baskin \& Baskin (1998) \\
\hline Galium aparine & Rubiaceae & $\mathrm{LF} / \mathrm{EC}$ & $10-17$ & $L>$ & & Baskin \& Baskin (1998) \\
\hline Galium aparine & Rubiaceae & $\mathrm{LF} / \mathrm{EC}$ & $20 / 15$ & $=$ & & Grime et al. (1981) \\
\hline Geoffroea decorticans & Fabaceae & $\mathrm{LN}$ & & & Sin pulpa & Cabello (1990) \\
\hline Geranium dissectum & Geraniaceae & Lfis/E & $20 / 15$ & & & Grime et al. (1981) \\
\hline Geranium molle & Geraniaceae & Lfis/E & $20 / 15$ & & & Grime et al. (1981) \\
\hline Geranium robertianum & Geraniaceae & Lfis/E & & $=$ & & Slade \& Causton (1979) \\
\hline Gomortega keule & Gomortegaceae & Lfis/E & & & & Donoso \& Escobar (1985) \\
\hline Hieracium aurantiacum & Asteraceae & $\mathrm{LF} / \mathrm{EF}$ & 22 & & & Stergios (1976) \\
\hline Holcus lanatus & Poaceae & SL & $20 / 16$ & $L>$ & & Grime et al. (1981) \\
\hline Hordeum mиrinum & Poaceae & SL & $25 / 10$ & & & Johnston et al. $(1981,1992)$ \\
\hline Ipomoea purpurea & Convolvulaceae & Lfis/E & $35 / 20$ & $=$ & & Baskin \& Baskin (1998) \\
\hline Jubaea chilensis & Palmaceae & $\mathrm{LF} / \mathrm{EC}$ & $35 / 25$ & & & Infante 1989, Cabello (1999) \\
\hline Lactuca serriola & Asteraceae & SL & $20 / 10$ & L> & & Baskin \& Baskin (1998) \\
\hline Lamium amplexicaule & Lamiaceae & $\mathrm{LF} / \mathrm{EC}$ & $15 / 6$ & L> & & Baskin \& Baskin (1981a) \\
\hline Lapsana communis & Asteraceae & $\mathrm{LF} / \mathrm{EC}$ & $15 / 10$ & $L>$ & & Baskin \& Baskin (1998) \\
\hline Lithrea caustica & Anacardiaceae & LfisF/E,EF & & & Digestión & $\begin{array}{l}\text { Cabello (1979), León-Lobos \& } \\
\text { Arroyo (1994) }\end{array}$ \\
\hline Lolium multiflorum & Poaceae & $\mathrm{LF} / \mathrm{EC}$ & $10 / 5$ & & & Young et al. (1975) \\
\hline Lolium rigidum & Poaceae & & $24 / 12$ & $L>$ & & Baskin \& Baskin (1998) \\
\hline Lotus corniculatus & Fabaceae & Lfis/E & $20 / 15$ & & & Grime et al. (1981) \\
\hline Luma apiculata & Myrtaceae & SL & & & & Cabello (1990) \\
\hline Luma chequen & Myrtaceae & SL & & & & Cabello (1990) \\
\hline Maytenus boaria & Celastracea & Lfis-F/EF,Em & 5 & & & $\begin{array}{l}\text { Hoffmann \& Kummerow (1962) } \\
\text { Cabello \& Camelio (1995) }\end{array}$ \\
\hline Medicago lupulina & Fabaceae & Lfis/E & $20 / 15$ & $=$ & & Grime et al (1981) \\
\hline Medicago sativa & Fabaceae & Lfis & $14-17$ & $=$ & & Baskin \& Baskin (1998) \\
\hline Melilotus alba & Fabaceae & Lfis/E & & & & Baskin \& Baskin (1998) \\
\hline Muehlenbeckia hastulata & Polygonaceae & Lfis/E & & & Golpe $\mathrm{T}^{\circ}$ & Muñoz \& Fuentes (1989) \\
\hline Myrceugenia correifolia & Myrtaceae & & 10 & & & Cabello et al. (2000) \\
\hline Onopordum acanthium & Asteraceae & $\mathrm{LF} / \mathrm{EF}$ & $30 / 20$ & $L>$ & & Baskin \& Baskin (1998) \\
\hline Oryza sativa & Poaceae & $\mathrm{LF} / \mathrm{EC}$ & 30 & & & Cohn \& Hughes (1981) \\
\hline Oxalis corniculta & Oxalidacea & SL & 17 & $\mathrm{~L}$ & & Baskin \& Baskin (1998) \\
\hline Panicum capillare & Poaceae & $\mathrm{LF} / \mathrm{EF}$ & $30 / 15$ & L> & & Baskin \& Baskin (1986) \\
\hline Panicum dichotomiflorum & Poaceae & $\mathrm{LF} / \mathrm{EF}$ & $35 / 20$ & L> & & Baskin \& Baskin (1983) \\
\hline Papaver dubium & Papaveraceae & $\mathrm{LMF} / \mathrm{EF}$ & 5 & & & Grime et al. (1981) \\
\hline Papaver rhoeas & Papaveraceae & $\mathrm{LMF} / \mathrm{EF}$ & $20 / 10$ & & & Baskin \& Baskin (1998) \\
\hline Pastinaca sativa & Apiaceae & LM & $30 / 15$ & $\mathrm{~L}>$ & & Baskin \& Baskin (1979b) \\
\hline Peumus boldus & Monimiaceae & Lfis/E & & & Golpe $\mathrm{T}^{\circ}$ & Muñoz (1986), Muñoz \& Fuentes (1989) \\
\hline Persea lingue & Lauraceae & SL & & & & Sáenz de Urtury (1986) \\
\hline Phacelia secunda & Hydrophyliaceae & $\mathrm{LF} / \mathrm{EF}$ & & & & Cavieres \& Arroyo (2000) \\
\hline
\end{tabular}


TABLA 1 (Continuación)

\begin{tabular}{|c|c|c|c|c|c|c|}
\hline Especie & Familia & L/Trat ${ }^{1}$ & Temp $^{2}$ & $\mathrm{Luz}^{3}$ & Otros $^{4}$ & Referencia \\
\hline Plantago lanceolata & Plantaginaceae & LF/EE & $30 / 20$ & $\mathrm{~L}$ & & Baskin \& Baskin (1998) \\
\hline Plantago major & Plantaginaceae & $\mathrm{LF} / \mathrm{EF}$ & $30 / 20$ & $\mathrm{~L}$ & & Baskin \& Baskin (1998) \\
\hline Роа аппиа & Poaceae & $\mathrm{LF} / \mathrm{EC}$ & 10,15 & & & Standifer \& Wilson (1988) \\
\hline Poa pratensis & Poaceae & $\mathrm{LF} / \mathrm{EF}$ & 10,15 & & & Baskin \& Baskin (1998) \\
\hline Polygonum aviculare & Polygonaceae & $\mathrm{LF} / \mathrm{EF}$ & $35 / 20$ & L> & & Baskin \& Baskin (1990b) \\
\hline Polygonum lapathifolium & Polygonaceae & $\mathrm{LF} / \mathrm{EF}$ & $30 / 10$ & $=$ & & Baskin \& Baskin (1998) \\
\hline Porlieria chilensis & Zygophyllaceae & $\mathrm{LN}$ & & & Digestión & Silva (2001) \\
\hline Portulaca oleracea & Portulacaceae & $\mathrm{LF} / \mathrm{EC}$ & $35 / 20$ & L> & & Baskin \& Baskin (1988) \\
\hline Prosopis chilensis & Mimosaceae & Lfis/E & & & & Cabello (1990) \\
\hline Psoralea glandulosa & Papilionaceae & SL & & & & Cabello (1990) \\
\hline Quillaja saponaria & Rosaceae & $\mathrm{LN}$ & $15-20$ & & & Muñoz \& Fuentes (1989), Wiberg (1990) \\
\hline Raphanus raphanistrum & Brassicaceae & $\mathrm{LF} / \mathrm{EC}$ & $30 / 20$ & O> & & Baskin \& Baskin (1998) \\
\hline Raphithamnus spinosus & Verbenaceae & $\mathrm{LN}$ & & & & Cabello (1990) \\
\hline Retanilla ephedra & Rhamnaceae & Lfis-F/E,EF & & & & Cabello (1990) \\
\hline Rumex acetosella & Polygonaceae & $\mathrm{LF} / \mathrm{EC}$ & $20 / 15$ & $=$ & & Grime et al. (1981) \\
\hline Rumex crispus & Polygonaceae & $\mathrm{LF} / \mathrm{EC}$ & $30 / 15$ & $\mathrm{~L}$ & & Baskin \& Baskin (1985) \\
\hline Rumex obtusifolius & Polygonaceae & SL & $30 / 20$ & L> & & Baskin \& Baskin (1998) \\
\hline Schinus molle & Anacardiaceae & $\mathrm{LN}$ & & & Digestión & Cabello 1990, Castro et al. (1994) \\
\hline Schinus polygamus & Anacardiaceae & Lfis/E & & & Golpe $\mathrm{T}^{\circ}$ & Muñoz \& Fuentes (1989) \\
\hline Senecio vulgaris & Asteraceae & LF & 10 & $\mathrm{~L}$ & & Baskin \& Baskin (1998) \\
\hline Setaria viridis & Poaceae & $\mathrm{LF} / \mathrm{EF}$ & 25 & & & Baskin \& Baskin (1998) \\
\hline Sisymbrium officinale & Brassicaceae & $\mathrm{LF} / \mathrm{EC}$ & & $\mathrm{L}$ & & Baskin \& Baskin (1998) \\
\hline Solanum nigrum & Solanaceae & $\mathrm{LF} / \mathrm{EF}$ & $30 / 10$ & L> & & Baskin \& Baskin (1998) \\
\hline Sophora macrocarpa & Papilionaceae & Lfis/E & & & & Cabello (1990) \\
\hline Sorghum halepense & Poacea & $\mathrm{LF} / \mathrm{EF}$ & $40 / 20$ & & & Baskin \& Baskin (1998) \\
\hline Spergula arvensis & Caryophyllaceae & $\mathrm{LF} / \mathrm{EF}$ & $27 / 19$ & & & Baskin \& Baskin (1998) \\
\hline Stellaria media & Caryophyllaceae & $\mathrm{LF} / \mathrm{EC}$ & $15 / 6$ & L> & & Baskin \& Baskin (1998) \\
\hline Taraxacum officinale & Asteraceae & SL & $10-18$ & L> & & Baskin \& Baskin (1998) \\
\hline Trevoa quinquenervia & Rhamnaceae & Lfis-F/E,EF & & & & Cabello (1990) \\
\hline Trevoa trinervis & Rhamnaceae & Lfis-F/E,EF & & & Golpe $\mathrm{T}^{\circ}$ & Muñoz \& Fuentes (1989), Cabello (1990) \\
\hline Trifolium glomeratum & Fabaceae & Lfis/E & $5-15$ & & & Olivares et al. (1990) \\
\hline Trifolium pratense & Fabaceae & Lfis/E & $20 / 15$ & $=$ & & Grime et al. (1981) \\
\hline Trifolium repens & Fabaceae & Lfis/E & $20 / 15$ & $=$ & & Grime et al. (1981) \\
\hline Trifolium subterraneum & Fabaceae & Lfis,F/ & $25 / 15$ & & & Baskin \& Baskin (1998) \\
\hline Tristerix aphyllus & Loranthaceae & & & & Digestión & Soto-Gamboa \& Bozinovic (2002) \\
\hline Verbascum thapsus & Scrophularaceae & $\mathrm{LF} / \mathrm{EF}$ & $35 / 20$ & L> & & Baskin \& Baskin (1981b) \\
\hline Verbena officinalis & Scrophularaceae & $\mathrm{LF} / \mathrm{EF}$ & $20 / 15$ & L> & & Grime et. al. (1981) \\
\hline Veronica arvensis & Scrophularaceae & $\mathrm{LF} / \mathrm{EC}$ & $15 / 6$ & $\mathrm{~L}$ & & Baskin \& Baskin (1998) \\
\hline Viola arvensis & Violaceae & $\mathrm{LF} / \mathrm{EC}$ & $15 / 6$ & $\mathrm{~L}$ & & Baskin \& Baskin (1995) \\
\hline Vicia hirsuta & Fabaceae & Lfis/E & $20 / 15$ & $=$ & & Grime et al. (1981) \\
\hline Vicia sativa & Fabaceae & Lfis/E & $20 / 15$ & $=$ & & Grime et al. (1981) \\
\hline Vulpia bromoides & Poaceae & SL & 15 & & & Johnston et al. (1989) \\
\hline
\end{tabular}

${ }^{1}$ L/Trat: tipo de latencia/tratamiento para desactivar latencia; Lfis: latencia física; LF: latencia fisiológica; LMF: latencia morfofisiológica; LM: latencia morfológica; Lfis-F: latencia física-fisiológica; LN: latencia no definida; SL: sin latencia innata. Los tipos de latencia siguen a Baskin \& Baskin (1989); E: escarificación ácida; Em: escarificación mecánica; EC: estratificación cálida; EF: estratificación fría

${ }^{2}$ Temp: temperatura óptima o con alto porcentaje de germinación $\left({ }^{\circ} \mathrm{C}\right)$

${ }^{3}$ Luz: requerimiento de luz para germinación; L>: germinación sensible a la luz blanca; O>: germinación inhibida por la luz blanca; L: germinación dependiente de la luz blanca; = germinación insensible a la luz.

${ }^{4}$ Otros: otros requerimientos para incrementar la germinación; Lav/NaCl: lavado con $\mathrm{NaCl}$; Sin pulpa: extracción manual de la pulpa; Sin arilo: extracción manual del arilo; Golpe $\mathrm{T}^{\circ}$ : golpe térmico $100{ }^{\circ} \mathrm{C}$ por 5 min; Digestión: digestión del fruto por frugívoros 
ción de semillas que requieren estratificación cálida y a la germinación de Erodium spp. que se han mantenido por menos de 2 años en un pequeño banco de semillas persistente. El acervo de semillas germinables queda prácticamente vacío al inicio de primavera y se colma rápidamente durante primavera-verano.

La fracción de semillas en el banco transitorio está constituida principalmente por semillas de hierbas y en menor proporción por especies leñosas. Para que las semillas de la mayoría de las hierbas introducidas escapen del banco transitorio hacia el acervo germinable requieren principalmente de una estratificación fría. Asimismo, para que las especies leñosas nativas en su mayoría escapen hacia la sección germinable requieren de una escarificación o abrasión de la testa.

Varios estudios sugieren que el banco de semillas persistente en Chile central es estructuralmente marginal (Armesto et al. 1995). Las semillas de las especies leñosas están ausentes del banco persistente y las semillas de hierbas están, pero serían minoritarias (Olivares et al. 1994), en una representación cuantitativa aún desconocida para el matorral. La mayoría de los bancos persistentes en vegetación mediterránea requieren de perturbaciones ocasionales e impredecibles para que sus semillas sean liberadas de la latencia. En Chile central estas perturbaciones no han sido identificadas, pero en la región árida y semiárida de Chile se ha detectado que muchas especies del banco persistente se liberan de su latencia debido al incremento de las precipitaciones por el fenómeno de El Niño (Gutiérrez et al. 2000, Gutiérrez 2001, Gutiérrez \& Meserve 2003).

Aunque todas las evidencias existentes para Chile central sugieren la presencia marginal de un banco persistente de semillas, su importancia como reservorio genético, poblacional y evolutivo en las especies nativas e introducidas está por estudiarse.

Las pérdidas del banco de semillas, aunque no son objeto de esta revisión, son producidas por patógenos, granívoros y factores de mortalidad de las semillas. Los estudios realizados hasta ahora con especies leñosas nativas del matorral (Bustamante et al. 1993, Bustamante \& Vásquez 1995) y de hierbas y leñosas nativas del bosque montano de la región mediterránea

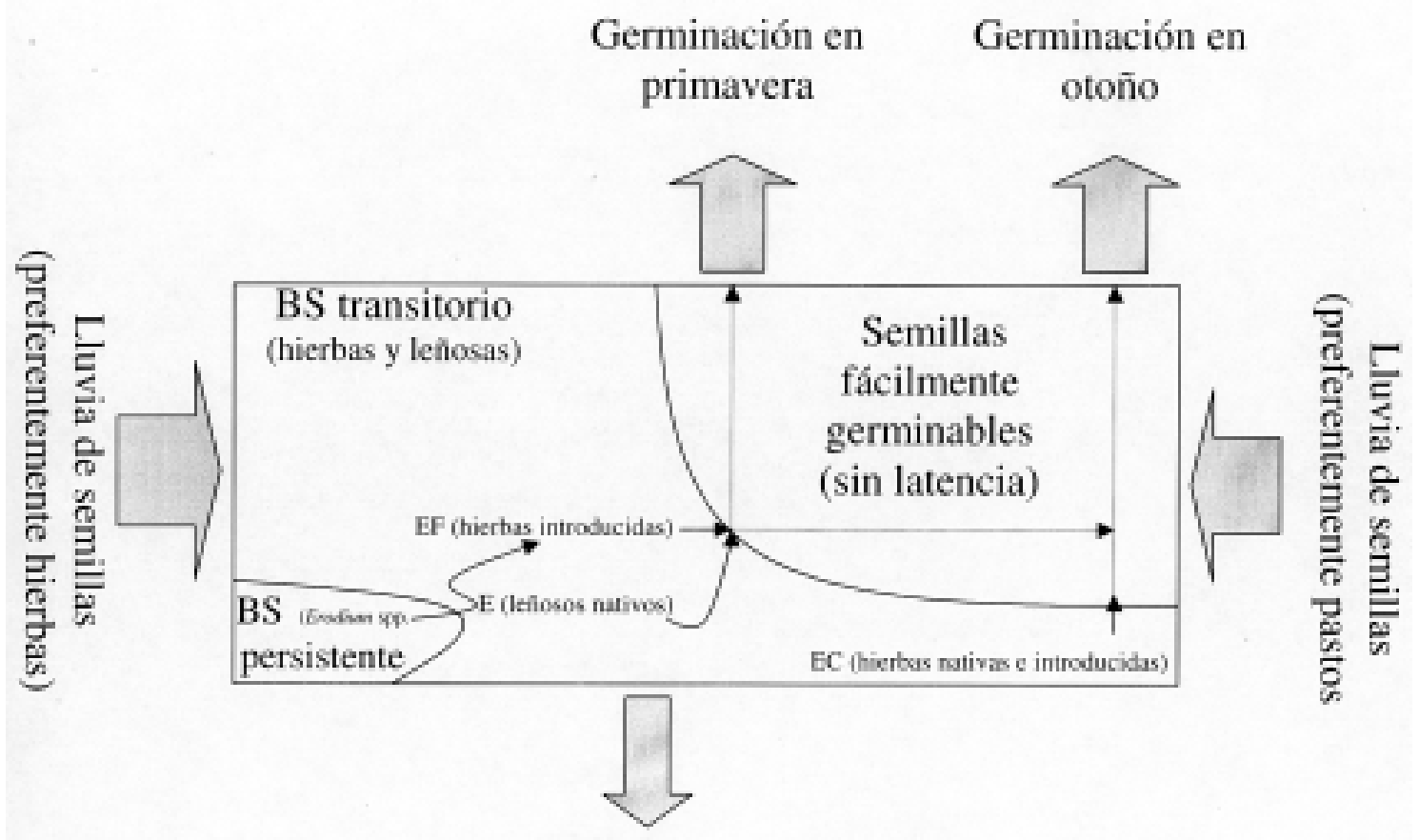

\section{Pérdidas}

Fig. 1: Modelo conceptual que representa las principales estructuras del banco de semillas en el matorral de Chile central: (BS) banco de semillas; (EF) estratificación fría de las semillas; (EC) estratificación cálida de las semillas; (E) escarificación de las semillas.

Conceptual model showing that main structures of the seed bank in the matorral of central Chile: (BS) seed bank; (EF) chilling of seeds; (EC) heat stratification of seeds; (E) seed scarification. 
(Figueroa et al. 2002) indican que por lo general la pérdida de semillas por efecto de los granívoros no sería dramática.

\section{AGRADECIMIENTOS}

Este estudio fue financiado por el proyecto FONDECYT-FONDAP 1501-0001. Durante la elaboración de esta revisión JA Figueroa fue apoyado por la Fundación AW Mellon y CASEB. Esta revisión recibió sugerencias de SA Castro, P León-Lobos y dos revisores anónimos.

\section{LITERATURA CITADA}

ACEITUNO P (1990) Anomalías climáticas en la región sudamericana durante los extremos de la oscilación austral. Revista Geofísica (Chile) 32: 65-78.

ARMESTO JJ, PE VIDIELLA, HE JIMÉNEZ (1995) Evaluating causes and mechanisms of succession in the Mediterranean regions in Chile and California. En: Arroyo MTK, PH Zedler \& MD Fox (eds) Ecology and biogeography of Mediterranean ecosystems in Chile, California, and Australia: 418433. Springer-Verlag, New York, New York, USA.

ARONSON J, A DEL POZO, C OVALLE, J AVENDAÑO, A LAVÍN \& M ETIENNE (1998) Land use changes and conflicts in central Chile. En: Rundel PW, G Montenegro \& FM Jaksic (eds) Landscape degradation and biodiversity in Mediterranean-type ecosystems: 155-168. SpringerVerlag, Berlin, Germany.

ARROYO MTK, LA CAVIERES, AM HUMAÑA \& C CASTOR (1999) Persistent seed bank and standing vegetation in a high alpine site in the central Chilean Andes. Oecologia 119: 126-132.

ARROYO MTK, C MARTICORENA, O MATTHEI, \& LA CAVIERES (2000) Plant invasions in Chile: present patterns and future predictions. En: Mooney $\mathrm{HA} \& \mathrm{RJ}$ Hobbs (eds) Invasive species in a changing world: 385-421. Island Press, Covelo, California, USA.

BASKIN JM \& CC BASKIN (1979a) Timing of seed germination in the weedy summer annual Euphorbia supina (maculata). Bartonia 46: 63-68.

BASKIN JM \& CC BASKIN (1979b) Studies on the autoecology and population biology of the weedy monocarpic perennial Pastinaca sativa. Journal of Ecology 67: 601-610.

BASKIN JM \& CC BASKIN (1980) Ecophysiology of secondary dormancy in seeds of Ambrosia artemisiifolia. Ecology 61: 475-480.

BASKIN JM \& CC BASKIN (1981a) Seasonal changes in germination responses of buried Lamium amplexicaule seeds. Weed Research 21: 299-306.

BASKIN JM \& CC BASKIN (1981b) Seasonal changes in germination responses of buried seeds of Verbascum thapsus and V. blattaria and ecological implications. Canadian Journal of Botany 59: 1769-1775.

BASKIN JM \& CC BASKIN (1983) Seasonal changes in germination responses of fall panicum to temperature and light. Canadian Journal of Plant Science 63: 973-979.

BASKIN JM \& CC BASKIN (1985) Does seed dormancy play a role in the germination ecology of Rumex crispus? Weed Science 33: 340-155.

BASKIN JM \& CC BASKIN (1986) Seasonal changes in germination responses of buried witchgrass (Panicum capillare) seeds. Weed Science 34: 22-24.

BASKIN JM \& CC BASKIN (1987) Temperature requirements for after-ripening in buried seeds of four summer annual weeds. Weed Research 27: 385-389.

BASKIN JM \& CC BASKIN (1988) Role of temperature in regulating the timing of germination in Portulaca oleraceae. Canadian Journal of Botany 66: 563-567.

BASKIN JM \& CC BASKIN (1989) Physiology of dormancy and germination in relation to seed bank ecology. En: Leck MA, VT Parker \& RL Simpson (eds) Ecology of soil seed bank: 231-256. Academic Press, San Diego, California, USA.

BASKIN JM \& CC BASKIN (1990a) Seed germination ecology of poison hemlock, Conium maculatum. Canadian Journal of Botany 68: 2018-2024.

BASKIN JM \& CC BASKIN (1990b) The role of light and alternating temperatures on germination of Polygonum aviculare seeds exhumed on various dates. Weed Research 30: 397-402.

BASKIN JM \& CC BASKIN (1995) Variation in the annual dormancy cycle in buried seeds of the weedy winter annual Viola arvensis. Weed Research 35: 353-362.

BASKIN CC \& JM BASKIN (1998) Seeds: Ecology, Biogeography, and Evolution of Dormancy and Germination. Academic Press. San Diego, California. $666 \mathrm{pp}$

BASKIN JM, CC BASKIN \& DM SPOONER (1989) Role of temperature, light and date seeds were exhumed from soil on germination of four wetland perennials. Aquatic Botany 35: 387-394.

BELL DT (1999) The process of germination in Australian species. Australian Journal of Botany 47: 475-517.

BOUCHER C \& EJ MOLL (1981) South Africa Mediterranean shrublands. En: di Castri F, GW Goodall \& RL Specht (eds) Ecosystems of the world, Volume 11: 233-248. Elsevier, Amsterdam, The Netherlands.

BUSTAMANTE RO \& RA VÁSQUEZ (1995) Granivoría en Cryptocarya alba (Mol.) Looser (Lauraceae): los efectos del tipo de hábitat y la densidad de semillas. Revista Chilena de Historia Natural 68: 117-122.

BUSTAMANTE RO, JA SIMONETTI \& JE MELLA (1992) Are foxes legitimate and efficient seed dispersers? A field test. Acta Oecologica 13: 203-208.

BUSTAMANTE RO, AA GREZ, JA SIMONETTI, RA VÁSQUEZ \& AM WALKOWIAK (1993) Antagonistic effect of frugivores on seeds of Cryptocarya alba (Mol.) Looser (Lauraceae): consequences on seedling recruitment. Acta Oecologica 14: 739-745.

BUSTAMANTE RO, A WALKOWIAK, CA HENRÍQUEZ \& I SEREY (1996) Bird frugivory and the fate of seeds of Cryptocarya alba (Lauraceae) in the Chilean matorral. Revista Chilena de Historia Natural 69: 357-363.

CABELLO A (1979) Estudio anatómico y de germinación en litre (Lithrea caustica (Mol.) H. et Arn.). Tesis de Ingeniería Forestal, Facultad de Ciencias Forestales, Universidad de Chile, Santiago, Chile. 108 pp.

CABELlO A (1990) Propagación de especies perteneciente a los bosques esclerófilos y espinosos de la zona central de Chile. Facultad de Ciencias Agrarias y Forestales, Departamento de Silvicultura, Universidad de Chile, Apuntes Docentes 3: 56-74.

CABELLO A (1999) Determinación del contenido de humedad y de la germinación de semillas de Jubaea chilensis en el vivero y en el laboratorio y de em- 
briones extirpados cultivados in vitro. Centro de Estudios de Semillas de Árboles Forestales (Chile) $\mathrm{N}^{\circ}$ 11. Universidad de Chile, Facultad de Ciencias Forestales, Departamento de Silvicultura. http:// uchile.cl/facultades/cs_forestales/cesaf

CABELLO A \& C BOTTI (1987) Estudios de germinación en Drimys winteri Forst. (Canelo). Ciencias Forestales (Chile) 5: 21-30.

CABELLO A \& ME CAMELIO (1995) Germinación de semillas y producción de plantas de Maitén (Maytenus boaria Mol). Centro de Estudios de Semillas de Árboles Forestales (Chile) $\mathrm{N}^{\circ} 6$. Universidad de Chile, Facultad de Ciencias Forestales, Departamento de Silvicultura. http://uchile.cl/faculta$\mathrm{des} / \mathrm{cs}$ forestales/cesaf.

CABELlO A \& A ALVEAR (1999) Temperatura de germinación en semillas de Acacia caven (espino). Centro de Estudios de Semillas de Árboles Forestales (Chile) $\mathrm{N}^{\circ} 10$. Universidad de Chile, Facultad de Ciencias Forestales, Departamento de Silvicultura. http://uchile.cl/facultades/cs_forestales/cesaf.

CABELLO A \& M ANSELMO (1999) Algunos factores que inciden en la germinación y producción de plantas de Beilschmiedia miersii (Belloto del Norte). Centro de Estudios de Semillas de Árboles Forestales (Chile) $\mathrm{N}^{\circ}$ 9. Universidad de Chile, Facultad de Ciencias Forestales, Departamento de Silvicultura. http://uchile.cl/facultades/cs_forestales/cesaf

CABELLO A, A ALVEAR \& M GONZÁLEZ (2000). Producción de plantas en el vivero Antumapu para la recuperación del bosque de Fray Jorge: Petrillo (Myrceugenia correifolia). Centro de Estudios de Semillas de Árboles Forestales (Chile) $\mathrm{N}^{\circ} 12$. Universidad de Chile, Facultad de Ciencias Forestales, Departamento de Silvicultura. http://uchile.cl/facultades/cs_forestales/cesaf

CASTRO SA, SI SILVA, PL MESERVE, JR GUTIÉRREZ, LC CONTRERAS \& FM JAKSIC (1994) Frugivoría y dispersión de semillas de pimiento (Schinus molle) por el zorro culpeo (Pseudalopex culpaeus) en el Parque Nacional Fray Jorge (IV Región, Chile). Revista Chilena de Historia Natural 67: 169-176.

CAVIERES LA (1999) Bancos de semillas persistentes: modelos de germinación retardada de semillas y su aplicación en ambientes alpinos. Revista Chilena de Historia Natural 72: 457-466.

CAVIERES LA \& MTK ARROYO (2000) Seed germination response to cold stratification period and termal regime in Phacelia secunda (Hydrophyllaceae): Altitudinal variation in the Mediterranean Andes of central Chile. Plant Ecology 149: 1-8.

CAVIERES LA \& MTK ARROYO (2001) Persistent soil seed banks in Phacelia secunda J.F. Gmel. (Hydrophyllaceae): experimental detection of variation along an altitudinal gradient in the Andes of central Chile. Journal of Ecology 88: 31-39.

CIPOLLINI ML \& DJ LEVEY (1997) Secondary metabolites of fleshy vertebrate-dispersed fruits: adaptive hypotheses and implications for seed dispersal. American Naturalist 150: 346-372.

COHN MA \& JA HUGHES (1981) Seed germination in red rice (Oryza sativa). I. Effect of temperature on dry-afterripening. Weed Science 29: 402-404.

COWLING RM, RUNDEL PW, LAMONT BB, ARROYO MK \& M ARIANOUTSOU (1996) Plant diversity in Mediterranean-climate regions. Trends in Ecology and Evolution 11: 362-366.

DI CASTRI F \& ER HAJEK (1976) Bioclimatología de Chile. Universidad Católica de Chile, Santiago, Chile. 128 pp.
DONOSO C \& A CABELLO (1978) Antecedentes fenológicos de germinación de semillas de especies leñosas chilenas. Ciencias Forestales (Chile) 1: 31-41.

DONOSO C \& B ESCOBAR (1985) Germinación de Gomortega keule (Mol.) Baillon. Bosque (Chile) 6: $120-122$

FERNÁNDEZ G (1978) Aumento de la germinación en Atriplex repanda. I. Tratamiento con ácido sulfúrico. Phyton 36: 36: 117-121.

FIGUEROA JA \& JJ ARMESTO (2001) Communitywide germination strategies in a temperate rainforest of southern Chile: ecological and evolutionary correlates. Australian Journal of Botany 49: 411-425.

FIGUEROA J, J ARMESTO \& JF HERNÁNDEZ (1996) Estrategias de germinación y latencia de semillas en especies del bosque templado de Chiloé, Chile. Revista Chilena de Historia Natural 69: 260-299.

FIGUEROA JA, AA MUÑOZ, J MELLA \& MTK ARROYO (2002) Pre and postdispersal seed predation in a Mediterranean-type climate montane sclerophyllous forest in central Chile. Australian Journal of Botany 50: 183-195.

FIGUEROA JA, S TEILLIER \& FM JAKSIC (en prensa) Composition, size and dynamics of the seed bank in a Mediterranean shrubland of Chile. Austral Ecology.

FUENTES ER (1990) Landscape change in Mediterraneantype habitats of Chile: patterns and processes. En: Zonneveld IS \& RTT Forman (eds) Changing landscapes: an ecological perspective: 165-190. Springer-Verlag, Berlin, Germany.

FUENTES ER, OTAÍZA RD, ALLIENDE MC, HOFFMANN AJ \& POIANI A (1984) Shrubs clumps of the Chilean matorral vegetation: structure and possible maintenance mechanisms. Oecologia 62: 405-411.

FUENTES ER, AJ HOFFMANN, A POIANI \& MC ALLIENDE (1986) Vegetation change in large clearing: patterns in the Chilean matorral. Oecologia 68: 358-366.

GAJARDO R (1994) La vegetación natural de Chile. Editorial Universitaria, Santiago, Chile. $165 \mathrm{pp}$.

GINOCCHIO R, M HOLMGREN \& G MONTENEGRO (1994) Effect of fire on plant architecture in Chilean shrubs. Revista Chilena de Historia Natural 67: 177-182.

GRIME JP, G MASON, AV CURTIS, J RODMAN, SR BAND, M MOWFORTH, AM NEAL \& S SHAW (1981) A comparative study of germination characteristics in a local flora. Journal of Ecology 69: 1017-1059.

GROVES RH (1986) Invasion of mediterranean ecosystems by weeds. En: Dell B, AJM Hopkins \& BB Lamont (eds) Resilience in Mediterranean-type ecosystems: 129-145. Dr. W Junk Publishers, Dordrecht, The Netherlands.

GULMON SL (1977) A comparative study of the grassland of California and Chile. Flora Bd 166 S: 261-278.

GUERRERO FP \& WA WILLIAMS (1975) Influence of temperature on the growth of Erodium botrys and Trifolium subterraneum. Crop Science 15: 553-556.

GUTIÉRREZ JR. (2001) Dynamics of ephemeral plants in the coastal desert of north-central Chile. En: Prakash I (ed) Ecology of deserts environments: 105-125. Scientific Publishers, India.

GUTIÉRREZ JR \& P MESERVE (2003) El Niño effects on the soil seed bank dynamics in north-central Chile. Oecologia 134: 511-517.

GUTIÉRREZ JR, L AGUILERA \& R MORENO (1988) The effects of variable regimes of temperature and 
light on the germination of Atriplex repanda seeds in the semiarid region of Chile. Revista Chilena de Historia Natural 61: 61-65.

GUTIÉRREZ JR, G ARANCIO \& FM JAKSIC (2000) Variation in vegetation and seed bank in a Chilean semi-arid community affected by ENSO 1997. Journal of Vegetation Science 11: 641-648.

HARPER JL (1977) Population biology of plants. Academics Press, London, United Kingdom. Xx pp.

HOFFMANN A \& J KUMMEROW (1962) Aspectos anatómicos, morfológicos y de la fisiología de germinación de semillas de Mytenus boaria Mol. Phyton (Argentina) 18: 51-56.

HOFFMANN AJ \& JJ ARMESTO (1995) Modes of seed dispersal in the Mediterranean regions in Chile, California, and Australia. En: Arroyo MTK, PH Zedler \& MD Fox (eds) Ecology and biogeography of Mediterranean ecosystems in Chile, California, and Australia: 289-310. Springer-Verlag, New York, New York, USA.

HOFFMANN AJ, S TEILLIER \& ER FUENTES (1989) Fruit and seed characteristics of woody species in mediterranean-type regions of Chile and California. Revista Chilena de Historia Natural 62: 43-60.

HOLMES PM \& RM COWLING (1997) Diversity, composition and guild structure relationships between soil-stored seed banks and mature vegetation in alien plant-invaded South African fynbos shrublands. Plant Ecology 133: 107-122.

HOLMGREN M (2002) Exotic herbivores as drivers of plant invasions and switch to ecosystem alternative states. Biological Invasions 4: 25-33.

INFANTE L (1989) Estudio de la germinación de la Palma Chilena (Jubaea chilensis (Mol.) Baillon). Tesis de Ingeniería Forestal, Facultad de Ciencias Agrarias y Forestales, Universidad de Chile, Santiago, Chile. 132 pp.

JAKSIC FM (2001) Spatiotemporal variation patterns of plants and animals in San Carlos de Apoquindo, central Chile. Revista Chilena de Historia Natural 74: 477-502.

JIMÉNEZ HE \& JJ ARMESTO (1992) Importance of the soil seed bank of disturbed sites in Chilean matorral in early secondary succession. Journal of Vegetation Science 3: 579-586.

JOHNSTON M, G FERNÁNDEZ \& A OLIVÁREZ (1989) Caracterización de la germinación de seis especies de una pradera anual mediterránea. Phyton (Argentina) 50: 109-117.

JOHNSTON M, A OLIVARES \& G FERNÁNDEZ (1992) Capacidad germinativa en especies de la pradera anual mediterránea. I. Efecto del tiempo transcurrido desde la cosecha. Phyton (Argentina) 53: $117-124$.

KEELEY JE (1991) Seed germination and life history syndromes in the California Chaparral. Botanical Review 57: 81-116.

KEELEY JE (1995) Seed-germination patterns in fireprone mediterranean-climate regions. En: Arroyo MTK, PH Zedler \& MD Fox (eds) Ecology and biogeography of Mediterranean ecosystems in Chile, California, and Australia: 289-310. SpringerVerlag, New York, New York, USA.

KEELEY JE \& CJ FOTHERINGHAM (1997) Trace gas emissions and smoke-induced seed germination. Science 276: 1248-1250.

KEELEY SC \& AW JOHNSON (1977) A comparison of the pattern of herb and shrub growth in comparable sites in Chile and California. American Midland Naturalist 97: 20-32.
LANDRUM LR (1981) A monograph of the genus Myrceugenia (Myrtaceae). Flora Neotropical Monography 29: 1-37.

LEÓN-LOBOS P \& MT KALIN-ARROYO (1994) Germinación de semillas de Lithrea caustica (Mol.) H. et A. (Anacardiaceae) dispersadas por Pseudalopex sp. (Canidae) en el bosque esclerófilo de Chile central. Revista Chilena de Historia Natural 67: 59-64.

LÓPEZ JH \& RB AVILEZ (1988) The pre-treatment of seeds of four Chilean Prosopis to improve their germination response. Seed Science \& Technology 16: 239-246.

MOHAMED-YASSEEN Y, SA BARRINGER, WE SPLITTSTOESSEER \& S COSTANZA (1994) The role of seed coats in seed viability. Botanical Review 60: 426-439.

MONTENEGRO $G, S$ TEILLIER, $P$ ARCE \& V POBLETE (1991) Introduction of plants into the mediterranean-type climate area of Chile. En: Groves H \& F di Castri (eds) Biogeography of Mediterranean invasions: 103-114. Cambridge University Press, New York, New York, USA.

MONTENEGRO G, M GÓMEZ, F DÍAZ \& $R$ GINOCCHIO (2002) Regeneration potential of Chilean matorral after fire: an update view. En: Veblen T, W Baker, G Montenegro \& $\mathrm{T}$ Swetnam (eds) Fire and climatic change in temperate ecosystems of the Western Americas: 375-403. Springer-Verlag, New York, New York, USA.

MORENO R, JR GUTIÉRREZ \& LE AGUILERA (1990) El efecto del $\mathrm{NaCl}$ en la germinación de Atriplex repanda de la región semiárida de Chile. Revista Chilena de Historia Natural 63: 61-68.

MUÑOZ M (1986) Cultivo de embriones y ensayo de germinación en boldo (Peumus boldus Mol.). Tesis de Ingeniería Forestal, Facultad de Ciencias Agrarias y Forestales, Universidad de Chile, Santiago, Chile. 209 pp.

MUÑOZ A \& A PÉREZ (1981) Factores que influyen en la producción de plantas de Acacia caven (Mol.) Hook et Arn. y Prosopis chilensis (Mol.) Stuntz. Tesis de Ingeniería Forestal, Facultad de Ciencias Agrarias, Universidad de Chile, Santiago, Chile. 88 pp.

MUÑOZ MR \& ER FUENTES (1989) Does fire induce shrub germination in the Chilean matorral? Oikos 56: $177-181$

MYERS N, MITTERMEIER RA, MITTERMEIER CG, DA FONSECA GAB \& KENT J (2000) Biodiversity hotspots for conservation priorities. Nature 403: 853-858.

NAVEH Z (1975) The evolutionary significance of fire in the Mediterranean region. Vegetatio 29: 199-208.

OLIVARES A, M JOHNSTON \& G FERNÁNDEZ (1990) Efecto de la temperatura en la germinación de siete especies de la pradera anual mediterránea y caracterización de su emergencia. Simiente (Chile) 60: 123-131.

OLIVARES A, M JOHNSTON \& X CONTRERAS (1994) Influencia del estrato arbóreo en la reserva de semillas del suelo. Simiente (Chile) 64: 248-253.

PAIS P (2000) Ensayo de germinación de Austrocedrus chilensis "ciprés de la cordillera". Centro de Estudios de Semillas de Árboles Forestales (Chile) $\mathrm{N}^{\circ}$ 12. Universidad de Chile, Facultad de Ciencias Forestales, Departamento de Silvicultura. http:// uchile.cl/facultades/cs_forestales/cesaf

PARKER VT \& VR KELLY (1989) Seed bank in California chaparral and other mediterranean climate shrublands. En: Leck MA, VT Parker \& RL Simpson (eds) Ecology of soil seed bank: 231-256. Academic Press, San Diego, California, USA. 
PARSONS RF (1981) Eucalyptus scrubs and shrublands. En: Groves RH (ed) Australian vegetation: 227-252. Cambridge University Press, Cambridge, United Kingdom.

PECO B, ORTEGA M \& C LEVASSOR (1998) Similarity between seed bank and vegetation in Mediterranean grassland: a predictive model. Journal of Vegetation Science 9: 815-828.

PIERCE S \& RM COWLING (1991) Dynamics of soilstored seed bank of six shrubs in fire-prone dune fynbos. Journal of Ecology 79: 731-747.

RAMÍREZ C, M ROMERO \& O HENRÍQUEZ (1980) Estudios de germinación en semillas de Mirtáceas chilenas. Bosque (Chile) 3: 106-114.

REES M (1993) Trade-off among dispersal strategies in British plants. Nature 366: 150-152.

ROBERTS EH (1973) Predicting the storage life of seeds. Seed Science \& Technology 1: 499-514.

RUNDEL PW (1998) Landscape disturbance in Mediterranean-type ecosystems: A review. En: Rundel PW, G Montenegro \& FM Jaksic (eds) Landscape degradation and biodiversity in Mediterranean-type ecosystems: 3-22 SpringerVerlag, Berlin, Germany.

RUSSI L, PS COCKS \& EH ROBERTS (1992) Seed bank dynamics in a Mediterranean grassland. Journal Applied Ecology 29: 763-771.

SÁENZ DE URTURY X (1986) Estudio anatómico y de germinación de lingue (Persea lingue Nees.) Tesis de Ingeniería Forestal, Facultad de Ciencias Agrarias y Forestales, Universidad de Chile, Santiago, Chile. $137 \mathrm{pp}$

SILVA S (2001) Ecología trófica y nutricional del zorro culpeo (Pseudalopex culpaeus): restricciones digestivas y energéticas asociadas a la frugivoría y sus efectos sobre la dispersión de semillas. Tesis de Doctorado, Facultad de Ciencias Biológicas, Pontificia Universidad Católica de Chile, Santiago, Chile. 199 pp

SLADE EA \& DR CAUSTON (1979) The germination of some woodland herbaceous species under laboratory conditions: A multifactorial study. New Phytologist 83: 549-557.

SOLANO F, JW SCHARADER \& HD COBLE (1976) Germination, growth, and development of spurred anoda. Weed Science 24: 574-578.

SOTO-GAMBOA M \& F BOZINOVIC (2002) Fruitdisperser interaction in a mistletoe- bird system: a comparison of two mechanisms of fruits processing on seed germination. Plant Ecology 159: 171-174.
STANDIFER LC \& PW WILSON (1988) A high temperature requirement for after ripening of imbibed dormant Poa annua L. seeds. Weed Research 28: 365-371.

STERGIOS BG (1976) Achene production, dispersal, seed germination, and seedling establishment of Hieracium aurantiacum in an abandoned field community. Canadian Journal of Botany 54: 1189-1197.

THOMPSON K \& JP GRIME (1979) Seasonal variation in the seed banks of herbaceous species in ten contrasting habitats. Journal of Ecology 67: 893-921.

THOMPSON PA, P NEWMAN \& PD KEEFE (1979) Germination of species of Alstroemeria L. Gartenbauwissenschaft 44: 97-102.

VALBUENA L \& L TRABAUD (2001) Contribution of the soil seed bank to post-fire recovery of a heathland. Plant Ecology 152: 175-183.

VAN STADEN J, NAC BROWN, AK JÄGER \& TA JOHNSON (2000) Smoke as a germination cue. Plant Species Biology 15: 167-178.

VÁZQUEZ-YANES C \& A OROZCO-SEGOVIA (1998) Physiological ecology of Mediterrenean seeds: link with Ex Situ conservation of plants. En: Rundel PW, G Montenegro \& FM Jaksic (eds) Landscape degradation and biodiversity in Mediterranean-type ecosistems: 265-272. Springer-Verlag, Berlin, Germany.

VENABLE DL (1989) Modeling the evolutionary ecology of seed banks. En: Leck MA, VT Parker \& RL Simpson (eds) Ecology of soil seed bank: 67-87. Academic Press, San Diego, California, USA.

VENABLE DL \& L LAWLOR (1980) Delayed germination and dispersal in desert annuals: escape in space and time. Oecologia 46: 272-282.

VIDIELLA P \& JJ ARMESTO (1989) Emergence of ephemeral plant species from soil samples of the Chilean coastal desert in response to experimental irrigation. Revista Chilena de Historia Natural 62: 99-107.

VILLAGRÁN CM (1995) Quaternary history of the mediterranean vegetation of Chile. En: Arroyo MTK, PH Zedler \& MD Fox (eds) Ecology and biogeography of Mediterranean ecosystems in Chile, California, and Australia: 3-20. Springer-Verlag, New York, New York, USA.

WIBERG S (1990) Influencia de algunos factores en la germinación de quillay (Quillaja saponaria Mol.) y producción de plantas en vivero. Tesis de Ingeniería Forestal, Facultad de Ciencias Agrarias y Forestales, Universidad de Chile, Santiago, Chile. 130 pp.

YOUNG JA, RA EVANS \& BL KAY (1975) Germination of Italian ryegrass seeds. Agronomy Journal 67: 386-389. 